Research Paper

\title{
Efficacy and Toxicity of IMRT-Based Simultaneous Integrated Boost for the Definitive Management of Positive Lymph Nodes in Patients with Cervical Cancer
}

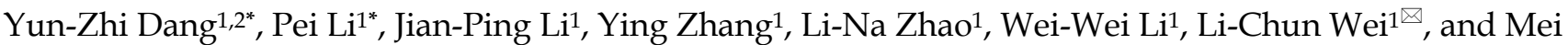 \\ Shi ${ }^{\boxplus}$ \\ 1. Department of Radiation Oncology, Xijing Hospital. The Fourth Military Medical University, Xi'an, Shaanxi, 710032, China \\ 2. State Key Laboratory of Cancer Biology, National Clinical Research Center for Digestive Diseases and Xijing Hospital of Digestive Diseases. The Fourth \\ Military Medical University, Xi' an, Shaanxi, 710032, China \\ *These authors contributed equally. \\ $\square$ Corresponding authors: Li-Chun Wei: weilichun@fmmu.edu.cn and Mei Shi: mshifmmu@yahoo.com \\ (c) Ivyspring International Publisher. This is an open access article distributed under the terms of the Creative Commons Attribution (CC BY-NC) license \\ (https://creativecommons.org/licenses/by-nc/4.0/). See http://ivyspring.com/terms for full terms and conditions.
}

Received: 2018.08.17; Accepted: 2019.01.04; Published: 2019.01.29

\begin{abstract}
Background: The optimal radiotherapy regimen for treating metastatic lymphadenopathy in patients with locally advanced cervical cancer remains controversial. This study aimed to investigate the clinical outcomes, as well as associated toxicities, of intensity-modulated radiotherapy (IMRT) with a simultaneous integrated boost (SIB) for pelvic and para-aortic lymph nodes (LNs).

Methods: Between 2011 and 2015, 74 patients with 2014 International Federation of Gynecology and Obstetrics stage IIB-IVB cervical cancer exhibiting pelvic or para-aortic LN involvement were examined. The pelvic field planning dose was 45-50 Gy in 25 fractions, and an SIB of $62.5 \mathrm{~Gy}$ in 25 fractions was delivered to positive LNs. Next, CT-guided brachytherapy was performed $24 \mathrm{~Gy}$ in 3 fractions to $42 \mathrm{~Gy}$ in 6 fractions once or twice weekly.

Results: The median follow-up duration was 36 (range: 3-62) months. The 3-year local control, distant metastasis-free survival, and overall survival rates were $91.7 \%, 75.7 \%$, and $71.4 \%$, respectively. No residual or recurrent LNs were detected. Six patients developed grade 3 acute gastrointestinal $(\mathrm{Gl})$ toxicity. Twenty-nine (39.2\%) and $3(4.1 \%)$ patients developed grade 3 and 4 hematological toxicities, respectively. Twenty patients $(28.5 \%)$ developed grade $\geq 2$ chronic $\mathrm{Gl}$ toxicity. Only 1 patient (1.4\%) experienced a grade 4 rectovaginal fistula, and 3 patients $(4.2 \%)$ developed grade 2 genitourinary toxicities. SIB to the LNs did not influence acute or chronic toxicity rates.
\end{abstract}

Conclusions: Our findings demonstrate that a dose of 62.5 Gy to positive LNs using the IMRT with SIB method can achieve excellent clinical outcomes with acceptable toxicity.

Key words: cervical cancer, lymph nodes, intensity-modulated radiotherapy simultaneous integrated boost

\section{Background}

Despite advances in radiotherapy and combined chemotherapy treatment modalities, the 5-year survival rate of patients with locally advanced cervical cancer remains approximately $70 \%$ [1]. The reported rate of lymph node (LN) involvement in patients with this disease ranges from $20 \%$ to $40 \%$; of these patients, approximately $50 \%$ may have additional para-aortic node (PAN) disease [2,3]. It is now universally recognized that in the absence of systemic metastasis, cervical cancer patients with LN involvement have a poor prognosis [4,5]. The 5-year overall survival (OS) of cervical cancer patients with and without evidence of LN metastasis is approximately $40 \%$ and $70 \%$, respectively [6]. 
High-risk LNs were previously demonstrated to be a predictor of poor prognosis [7]; furthermore, cervical cancer patients with PAN involvement have poorer prognoses $[8,9]$, and for such patients, extended field radiotherapy with concurrent cisplatin-based chemotherapy is the standard treatment.

The optimal treatment for bulky lymphadenopathy remains controversial although a high radiation dose is considered suitable for achieving pelvic or para-aortic LN control [10]. Definitive radiation therapy for the primary tumor is completed with a combination of external beam radiotherapy (EBRT) and brachytherapy boost, with doses that usually exceed 70 Gy (biologically equivalent dose of $2 \mathrm{~Gy}$ fractions [EQD 2$])$. Such dose levels can achieve excellent local disease control with acceptable toxicity. However, owing to the high rate of such toxicities, the conventional 3-4 field-box EBRT technique is unable to deliver sufficient doses to achieve locoregional LN control. The dose recommendation for positive LNs remains unknown and varies between 55 and $60 \mathrm{~Gy}$ [11].

Intensity-modulated radiotherapy (IMRT) can provide a more conformal dose distribution while reducing the absorbed dose to organs at risk (OARs). Furthermore, IMRT can deliver different doses to various regions of the irradiated volume via a simultaneous integrated boost (SIB). Although some studies have demonstrated the effectiveness of IMRT against cervical cancer [12], limited clinical data on the use of boost from EBRT to metastatic nodes are available [13].

Owing to the many advantages of both SIB-IMRT techniques, we developed a protocol for treating positive LNs that were detected using computed tomography (CT) and magnetic resonance imaging (MRI). This study aimed to determine whether SIB-IMRT to involved pelvic and para-aortic LNs during definitive chemoradiotherapy for locally advanced cervical cancer produces optimal clinical outcomes. Furthermore, this study aimed to evaluate the adverse effects of this treatment approach.

\section{Methods}

\section{Patient characteristics}

Between January 2011 and December 2015, 74 patients with 2014 International Federation of Gynecology and Obstetrics stage IIB-IVB cervical cancer who underwent definitive radiotherapy with IMRT-based SIB for positive LNs at the Department of Radiation Oncology of Xijing Hospital, the Fourth Military Medical University were included. All patients had either positive pelvic or para-aortic metastatic LNs. Patients with stage IVB cervical cancer were included only if they had para-aortic LN metastases, while those with metastasis to other sites, such as the lung and liver, were excluded.

Prior to radiotherapy, all patients underwent physical examination, chest radiography or $\mathrm{CT}$, cervical biopsy, abdominal CT, pelvic imaging with MRI and CT (except for patients who were medically ineligible), and measurement of squamous cell carcinoma antigen levels. Any LN that had a minimal axial diameter of $\geq 10 \mathrm{~mm}$ or exhibited central necrosis was defined as positive. The study was approved by the local Institutional Review Board, and informed consent was obtained from all included patients.

\section{Treatment}

All patients received EBRT to the pelvis via IMRT using the Eclipse version 8.9 treatment planning system (Varian Medical, Palo Alto, CA). IMRT was delivered with 6-MV photon beams generated from the Varian IX linear accelerator equipped with a 120-leaf Millennium multi-leaf collimator. Patients were treated using a double-arc volumetric-modulated arc radiotherapy plan or an IMRT plan with 9 coplanar fields using equally spaced gantry angles. An image was obtained from the CT simulator for treatment planning of all patients using serial 5-mm slices from the renal hilus to $3 \mathrm{~cm}$ below the ischial tuberosity; furthermore, for patients with stage IVB cervical cancer, the CT simulator initiated from the upper pole of the left kidney. The gross tumor volume (GTV) was defined as the cervical tumor; GTV-N was defined as pelvic or para-aortic LNs $\geq 1 \mathrm{~cm}$ in size. For patients with stages IIB-IVA cancer, the clinical target volume included the gross tumor, cervix, uterus, parametria, the upper part of the vagina to $3 \mathrm{~cm}$ below the level of tumor invasion, and the regional (common, external, internal iliac, obturator, and presacral) LNs. For patients with stage IVB cancer, the clinical target volume started at the left renal veins levels and included the entire para-aortic nodal region. According to the International Multi-Center Phase II Clinical Trial (Intertecc-2) [14], which we are a partner of, the planning target volumes (PTVs) were generally delineated by adding the following margins to the CTVs: a $15-\mathrm{mm}$ margin around the gross cervical tumor and the cervix and uterus, $10-\mathrm{mm}$ margins around the vagina and parametria, and $7-\mathrm{mm}$ margins around the regional LNs $(10 \mathrm{~mm}$ of the LNs were positive). The planning dose was $45-50$ Gy in 25 fractions for the pelvic field, and the SIB was 62.5 Gy in 25 fractions $\left(\mathrm{EQD}_{2}=65 \mathrm{~Gy}\right)$ for the positive LNs. Weekly on-line imaging was performed. Based on the changes observed, secondary CT simulation and radiotherapy planning will be conducted if necessary. 
Chemotherapy comprised weekly cisplatin (40 $\left.\mathrm{mg} / \mathrm{m}^{2}\right)$ or tri-weekly docetaxel $\left(60 \mathrm{mg} / \mathrm{m}^{2}\right)$ and cisplatin $\left(60 \mathrm{mg} / \mathrm{m}^{2}\right)$; both were administered during radiotherapy. Moreover, chemotherapy was withheld under the following conditions: (a) a white blood cell count $<2.0 \times 10^{3} / \mu \mathrm{L}$, (b) an absolute neutrophil count $<1.5 \times 10^{3} / \mu \mathrm{L}$, and/or (c) a platelet count $<7.5 \times 10^{4} / \mu \mathrm{L}$. When chemotherapy was delayed owing to grade 3 or 4 toxicity, the patient was reexamined after 1 week.

After completing EBRT, intracavity, interstitial, or combined intracavity/interstitial brachytherapy was performed using a CT-guided procedure. Based on the CT-standardized contour report [15], reference MR image, and Gynecological GEC-ESTOR working group recommendations [16, 17], high-risk CTVs, intermediate -risk CTVs, and OARs (intestine, rectum, sigmoid, and bladder) were contoured using CT. Patients were treated with a high-dose-rate iridium-192 source according to a treatment planning system (Nucletron Systems, Veenendaal, Netherlands). The prescribed dose was 24 Gy in 3 fractions to $42 \mathrm{~Gy}$ in 6 fractions once or twice weekly. The median treatment time was 59 (range: 38-100) days.

We calculated the $\mathrm{EQD}_{2}$ according to the linear-quadratic model for incomplete sublethal damage repair [18]. The $\mathrm{EQD}_{2}$ used the linear quadratic model with $\alpha / \beta=10$ Gy for the tumor and $\alpha / \beta=3$ Gy for OARs.

\section{Follow-up and toxicity}

Patients were followed up first 1 month and were subsequently examined every 3-6 months for the first 2 years, then every 6 months thereafter. Pelvic examinations, CT or MRI, and ultrasonography were performed at every visit. Suspected persistent or recurrent disease was based on physical or radiographic examinations or on pathological confirmation (which was preferred). Acute or chronic toxicities, including hematologic, gastrointestinal (GI), and genitourinary (GU) toxicities, were graded by the treating physician according to Radiation Therapy Oncology Group (RTOG) criteria.

\section{Statistical analysis}

The endpoints were local control rate (LC), distant metastasis-free survival (DFS), and OS. The time intervals for LC, DFS, and OS were calculated from the date of completion of radiotherapy to the date of the respective event or the last follow-up visit. Treatment failures were classified according to the site(s) of the first tumor relapse and were defined as local (cervix, vagina, and parametria), pelvic node, or distant metastases. For patients with stage IIB-IVA cancer, distant metastasis included peritoneal spread or involvement of the supraclavicular mediastinal, paraaortic lymph nodes, lung, liver, and bone. Furthermore, for patients with 2014 FIGO stage IVB cancer, since all of the patients with stage IVB cancer had paraaortic lymph nodes metastasis, we defined patients with metastasis to other sites, except paraaortic metastasis, as distant failure. Patient characteristics and toxicity were evaluated using descriptive summary statistics. Survival analyses were conducted using the Kaplan-Meier method. Data measurements, including dose-volume histogram parameters, were compared using analysis of variance or Student's $t$-test. Statistical analysis was conducted using SPSS version 18.0 (SPSS, Chicago, IL, USA). A p-value $<0.05$ was considered statistically significant.

\section{Results}

\section{Patient characteristics}

The patient and tumor characteristics are summarized in Table 1 . The median patient age was 51 (range: 32-76) years. All patients had pelvic LN involvement: 40 had only clinical pelvic LN involvement, whereas 34 had both clinical pelvic and para-aortic positive LNs. The short diameter of the positive LNs was $1-2 \mathrm{~cm}$ in 66 patients $(89.2 \%)$ and $>2$ $\mathrm{cm}$ in $8(10.8 \%)$. The tumors in all patients had squamous cell carcinoma histology. Forty-two patients received $40 \mathrm{mg} / \mathrm{m}^{2}$ of concurrent weekly cisplatin, 25 received triweekly docetaxel plus cisplatin, and 7 did not undergo concurrent chemotherapy.

Table 1. Patient and tumor characteristics

\begin{tabular}{ll}
\hline Characteristic & Value (n=74) \\
\hline Age at diagnosis (years) & Median: 51 (range: 32-76) \\
$\begin{array}{l}\text { Tumor diameter }(\mathbf{c m}) \\
\text { FIGO stage }\end{array}$ & Mean: $4.7(4.7 \pm 1.8)$ \\
IIB & $10(13.5 \%)$ \\
IIIA & $2(2.7 \%)$ \\
IIIB & $28(37.8 \%)$ \\
IVB* & $34(46 \%)$ \\
Histological type & \\
Squamous carcinoma & $100(100 \%)$ \\
Chemotherapy & \\
Weekly cisplatin & $42(56.8 \%)$ \\
Triweekly DP & $25(33.8 \%)$ \\
No chemotherapy & $7(9.4 \%)$ \\
Shortest diameter of LN & Median $1.3 \mathrm{~cm}(1.0-3.5)$ \\
Number of LN & Median $3(1-7)$ \\
Volume of LN & Median $10.42 \mathrm{~cm}^{3}(1.2-66.49)$ \\
Follow-up period (months) & Median: $36($ range:3- 62$)$ \\
Treatment period (days) & Median $59(42-88)$ \\
\hline *Among patients with stage IVB cancer, those with only para-aortic LN metastasis \\
were included.
\end{tabular}




\section{Clinical results}

The median follow-up duration was 36 (range: 3-62) months, by the end of which 20 patients died (19 owing to cancer and 1 owing to cerebral thrombosis). Pelvic recurrence was detected in 6 patients, and 19 experienced distant metastasis. Of the 6 patients with local recurrence, 5 had a recurrence in the cervix and 1 in the parametrium. No residual or recurrent LNs were detected. The 3-year LC, DFS, and OS rates were $91.7 \%, 75.7 \%$, and $71.4 \%$, respectively (Figure $1 \mathrm{a}$ ).

The main mode of failure for the 34 patients with stage IVB cervical cancer was distant metastasis; only 1 patient experienced local failure and 11 experienced distant metastasis. Among patients with stage IIB-IIIB cancer and those with stage IVB cervical cancer, the 3 -year LC rates were $90 \%$ and $94.1 \%$, respectively $(\mathrm{p}=0.504)$, the 3 -year DFS rates were $82.5 \%$ and $64.7 \%$, respectively $(p=0.04)$ (Figure $1 b)$, and the 3-year OS rates were $82.5 \%$ and $61.8 \%$, respectively $(p=0.033)$ (Figure 1c).

\section{Prognostic factors}

Clinical and treatment parameters, as well as LN characteristics, such as volume and number, were analyzed. As shown in Table 2, a higher positive LN number, higher tumor stage, and omission of chemotherapy were significantly correlated with poorer OS in the univariate analysis. Only a higher tumor stage was associated with a shorter DFS, while forgoing chemotherapy predicted poorer LC.

The results of the multivariate analysis are shown in Table 3. LN number $>4 \quad(p=0.011,95 \%$ confidence interval [CI]: 1.346-10.015), stage IVB cervical cancer with PAN involvement $(\mathrm{p}=0.038,95 \%$ CI: 1.065-9.211), and forgoing chemotherapy $(\mathrm{p}=0.001$, 95\% CI: 1.065-9.211) were predictive of poorer OS rates. Patients who did not undergo chemotherapy had poorer DFS rates ( $p=0.014,95 \%$ CI: 0.041-0.692) and worse LC rates ( $\mathrm{p}=0.044,95 \%$ CI: $0.011-0.936$ ).
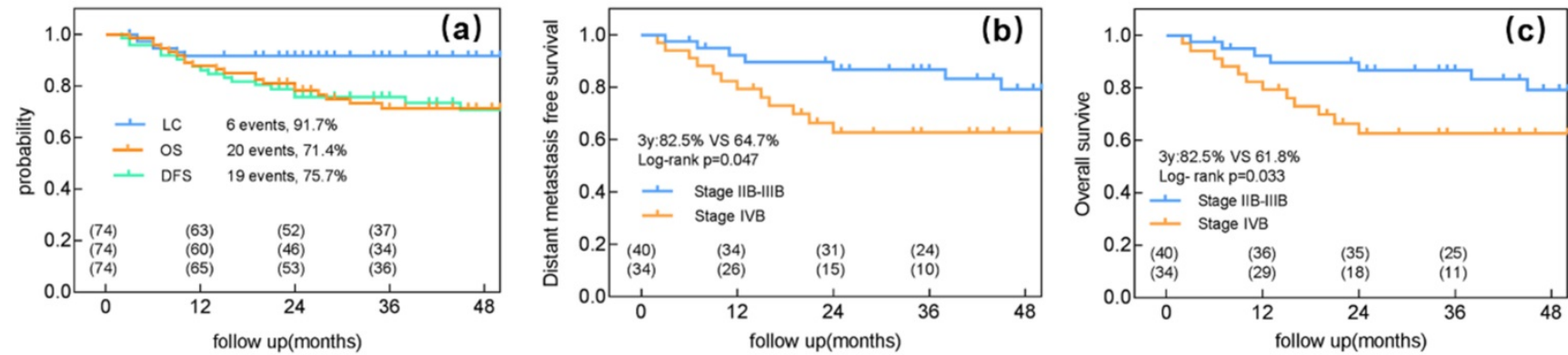

Figure 1. Clinical outcomes of intensity-modulated radiotherapy with a simultaneous integrated boost for the definitive management of node-positive cervical cancer. (a) Local control (LC), distant metastasis-free survival (DFS), and overall survival (OS) for all patients. (b) DFS for patients with either stage IIB-IIIB or IVB cervical cancer. (c) OS for patients with either FIGO stage IIB-IIIB or IVB cervical cancer.

Table 2. Univariate analysis of factors influencing OS, DFS, and LC

\begin{tabular}{|c|c|c|c|c|c|c|c|}
\hline Factors & No. of patients & 3-year OS (\%) & $p$ & 3-year DFS (\%) & $p$ & 3-year LC (\%) & $p$ \\
\hline \multicolumn{8}{|l|}{ Age (years old) } \\
\hline$\leq 50$ & 36 & 77.8 & 0.353 & 75 & 0.831 & 94.4 & 0.452 \\
\hline$>50$ & 38 & 68.4 & & 73.7 & & 89.5 & \\
\hline \multicolumn{8}{|l|}{ LN characteristics } \\
\hline $\mathrm{LN}$-volume $\leq 10 \mathrm{~cm}^{3}$ & 36 & 77.8 & 0.307 & 80.6 & 0.168 & 97.2 & 0.093 \\
\hline $\mathrm{LN}$-volume $>10 \mathrm{~cm}^{3}$ & 38 & 68.4 & & 68.4 & & 86.8 & \\
\hline LN-number $\leq 4$ & 52 & 82.7 & 0.02 & 78.8 & 0.120 & 94.2 & 0.220 \\
\hline LN-number $>4$ & 21 & 52.4 & & 66.7 & & 85.7 & \\
\hline \multicolumn{8}{|l|}{ Tumor stage } \\
\hline IIB-IIIB & 40 & 82.5 & 0.033 & 82.5 & 0.047 & 90 & 0.504 \\
\hline IVB & 34 & 64.7 & & 64.7 & & 94.1 & \\
\hline \multicolumn{8}{|l|}{ Chemotherapy } \\
\hline $\mathrm{NO}$ & 7 & 42.9 & 0.012 & 57.1 & 0.074 & 71.4 & 0.012 \\
\hline yes & 67 & 76.1 & & 76.1 & & 94 & \\
\hline \multicolumn{8}{|c|}{ Radiotherapy duration } \\
\hline$\leq 8$ weeks & 34 & 76.5 & 0.525 & 79.4 & 0.335 & 94.1 & 0.48 \\
\hline$>8$ weeks & 40 & 70 & & 70 & & 90 & \\
\hline \multicolumn{8}{|l|}{ Tumor diameter } \\
\hline$\leq 4$ & 46 & 67.4 & 0.221 & 76.1 & 0.674 & 91.3 & 0.763 \\
\hline$>4$ & 28 & 82.1 & & 71.4 & & 92.9 & \\
\hline
\end{tabular}

OS, overall survival; DFS, disease-free survival; LC, local control; LN, lymph node. 
Table 3. Multivariate analysis of factors influencing OS, DFS, and LC

\begin{tabular}{|c|c|c|c|c|c|c|}
\hline Factors & OS $(95 \% \mathrm{CI})$ & $p$ & DFS (95\% CI) & $p$ & LC (95\% CI) & $p$ \\
\hline \multicolumn{7}{|l|}{ LN characteristics } \\
\hline $\mathrm{LN}$-volume $\leq 10 \mathrm{~cm}^{3}$ & $1.310(0.477-3.595)$ & 0.601 & $1.868(0.667-5.235)$ & 0.234 & $7.488(0.578-97.039)$ & 0.123 \\
\hline \multicolumn{7}{|l|}{ LN-volume $>10 \mathrm{~cm}^{3}$} \\
\hline LN-number $\leq 4$ & $3.671(1.346-10.015)$ & 0.011 & $0.824(0.310-2.190)$ & 0.698 & $1.259(0.181-8.777)$ & 0.816 \\
\hline \multicolumn{7}{|l|}{ LN-number $>4$} \\
\hline \multicolumn{7}{|l|}{ Tumor stage } \\
\hline IIB-IIIB & $3.132(1.065-9.211)$ & 0.038 & $2.783(0.983-7.878)$ & 0.054 & $0.723(0.109-4.785)$ & 0.736 \\
\hline \multicolumn{7}{|l|}{ IVB } \\
\hline \multicolumn{7}{|l|}{ Chemotherapy } \\
\hline $\mathrm{NO}$ & $0.108(0.029-0.406)$ & 0.001 & $0.168(0.041-0.692)$ & 0.014 & $0.102(0.011-0.936)$ & 0.044 \\
\hline \multicolumn{7}{|l|}{ Yes } \\
\hline \multicolumn{7}{|l|}{ Tumor diameter } \\
\hline$\leq 4$ & $0.884(0.349-2.242)$ & 0.796 & $0.824(0.310-2.910)$ & 0.698 & $2.151(0.362-12.8)$ & 0.40 \\
\hline$>4$ & & & & & & \\
\hline
\end{tabular}

Table 4. Acute and late treatment-related toxicities

\begin{tabular}{|c|c|c|c|c|c|c|c|c|c|c|}
\hline \multirow{3}{*}{ Toxicity } & \multicolumn{5}{|c|}{ Acute Toxicities } & \multicolumn{5}{|c|}{ Chronic Toxicities } \\
\hline & \multicolumn{5}{|c|}{ Grade $(\%)$} & \multicolumn{5}{|c|}{ Grade (\%) } \\
\hline & 0 & 1 & 2 & 3 & 4 & 0 & 1 & 2 & 3 & 4 \\
\hline$\overline{\mathrm{GU}}$ & $59(82.4)$ & 15(17.6) & 0 & 0 & 0 & $50(67.5)$ & $21(28.3)$ & $3(4.2)$ & 0 & 0 \\
\hline GI & $10(13.5)$ & $28(37.9)$ & $30(40.5)$ & $6(8.1)$ & 0 & $30(40.5)$ & $23(31.0)$ & $17(23)$ & $3(4.1)$ & $1(1.4)$ \\
\hline Hematologic & $8(10.8)$ & $4(5.4)$ & $30(40.5)$ & $29(39.2)$ & $3(4.1 \%)$ & - & - & - & - & - \\
\hline
\end{tabular}

$\mathrm{GU}$, genitourinary; GI, gastrointestinal.

\section{LN SIB and toxicity}

Acute toxicity was assessed weekly during radiotherapy. Thirty $(40.5 \%)$ and $6(8.1 \%)$ patients developed grade 2 and 3 GI toxicity, respectively, whereas $29(39 \%)$ and $4(5.4 \%)$ patients developed grade 3 and 4 hematological toxicity, respectively. Twenty patients $(28.5 \%)$ developed grade $\geq 2$ chronic GI toxicity, with $3(4.1 \%)$ experiencing grade 3 symptoms. All grade $\geq 2$ chronic GI toxicities occurred in the rectum. Patients with grade 2 late side effects (LSEs) experienced similar symptoms and had intermittent bloody stool; most of these LSEs were treated with mesalazine. All events reported in patients with grade 3 LSEs were continuous bloody stool that required red blood cell transfusion. Only 1 patient experienced a grade 4 rectovaginal fistula $(1.4 \%)$. Moreover, 60 patients $(81 \%)$ reported no small bowel morbidity at any time during the follow-up period (grade 0), whereas 14 patients (19\%) experienced grade 1 LSEs. The most common events reported were slight diarrhea and discontinuous abdominal pain.

In this study, the mean small bowel volumes receiving 62.5 and 60 Gy were $7.13 \pm 15.8 \mathrm{~cm}^{3}$ and $11.8 \pm 21.1 \mathrm{~cm}^{3}$, respectively. There was no significant relationship between acute GI toxicity and the volume of the small bowel receiving 62.5 and $60 \mathrm{~Gy}(\mathrm{p}=0.89$ and $\mathrm{p}=0.702$, respectively). Moreover, the mean rectal volumes receiving 62.5 and 60 Gy were $0.18 \pm 0.81 \mathrm{~cm}^{3}$ and $2.1 \pm 0.44 \mathrm{~cm}^{3}$, respectively, with no significant relationship between rectal LSEs and the mean volume of the rectum receiving either 62.5 or $60 \mathrm{~Gy}$ $(p=0.37$ and $p=0.59$, respectively). The mean bladder volume receiving 62.5 or $60 \mathrm{~Gy}$ was $1.8 \pm 3.6$ and $4.0 \pm 7.5 \mathrm{~cm}^{3}$, respectively.

\section{Discussion}

Studies investigating the optimal dose for metastatic LNs arising from cervical cancer are scarce, particularly those regarding IMRT-based SIB for positive LNs. The present study demonstrated that synchronous boosts with EBRT or extended field-EBRT result in favorable control rates for pelvic and para-aortic LNs and are safe for cervical cancer patients with clinically positive LNs. All patients completed EBRT using an IMRT method, and the dose to positive LNs was boosted simultaneously using IMRT-based SIB. Our study revealed excellent clinical outcomes in terms of the 3-year LC, DFS, and OS.

A higher radiation dose more effectively controls $\mathrm{LN}$, although this notion remains to be properly validated. In a study of 48 patients with cervical cancer who received a median 63 Gy to gross LNs, Yoon et al [19] found that 37 patients (77\%) experienced a complete metabolic response in their LNs on positron emission tomography (PET)/CT imaging. In the RTOG 0116 trial [20], where a mean dose of 55.7 Gy was used, a complete response was documented in $60 \%$ and $71 \%$ of pelvic and para-aortic LNs, respectively. Using PET/CT, Rash et al [21] observed a significantly greater decrease in the standard uptake value of $18 \mathrm{~F}$-fluorodeoxyglucose in the LNs of patients receiving $\geq 54$ Gy than in those receiving $<54$ Gy $(p=0.006)$, and they recommended that the involved LNs should be treated with at least 54 Gy. 
Cihoric et al [22] found that 10 patients with positive LNs who received SIB with 62 Gy experienced no relapse. Moreover, Grigsby et al [23] found that a mean dose of 66.9 Gy resulted in a control rate of $100 \%$ (all of 21 patients) for PET/CT-positive LNs that were 1-2 $\mathrm{cm}$ in size; a mean dose of $69.4 \mathrm{~Gy}$ resulted in a control rate of $86.6 \%$ (13/15 patients) for PET/CT-positive LNs that were $2-3 \mathrm{~cm}$ in size. In our study, we prescribed a much higher radiation dose (62.5 Gy, EQD2 = 65 Gy) for patients with positive pelvic or para-aortic LNs, which resulted in no residual or recurrent LNs. Moreover, the smallest diameter of LNs was $1-2 \mathrm{~cm}$ in 66 patients $(89.1 \%)$ and $>2 \mathrm{~cm}$ in only 8 patients $(20.9 \%)$. These findings suggest that a radiation dose of $62.5 \mathrm{~Gy}\left(\mathrm{EQD}_{2}=65 \mathrm{~Gy}\right)$ is sufficient to control positive LNs.

Our multivariate analysis showed that an LN number $>4$ and a disease stage of IVB with PAN involvement were predictors of poorer OS, whereas a para-aortic LN involvement suggested a worse DFS $(p=0.054)$. Distance metastasis was the main failure pattern for patients with stage IVB cervical cancer with PAN involvement, and thus, a more intense, comprehensive treatment may be appropriate for such patients. The higher 3-year DFS of patients with stage IVB cancer in our study compared with that in previous studies could be due to the difference in the sample selection criteria. Because stage IVB cervical cancer patients with only para-aortic lymph nodes were included in our study and those with metastasis to other sites, such as the lung and liver, were excluded. Moreover, another reason could be the short size of lymph nodes was relatively small (range, $1.0-3.5 \mathrm{~cm}$ ).

The main concern when escalating doses is the increase in the incidence of serious acute adverse events. Several dosimetric studies have evaluated the advantages of IMRT for cervical cancer in terms of a high-dose gradient around the target volume. Forrest et al [24] compared conventional whole pelvis radiotherapy to IMRT, and in most of their patients, they found a $>20 \%$ reduction in the volume of OARs receiving at least 50 Gy with the latter method. Portelance et al ${ }^{[25]}$ also reported that IMRT produces a $30-70 \%$ reduction in the doses to OARs compared with conventional EBRT. In our study, 6 patients $(8.1 \%)$ developed grade 3 GI toxicity, whereas 29 $(39 \%)$ and $4(5.4 \%)$ patients developed grade 3 and 4 hematological toxicities, respectively. No patients required an interruption in radiotherapy because of acute radiation side effects.

Late toxicities of the proposed treatment technique are also an important concern. In our study, $28.5 \%$ and $4.1 \%$ of patients experienced grade 2 and 3 rectal LSEs, respectively. Only 1 patient experienced grade 4 LSEs. Moreover, $4.2 \%$ of the patients developed grade 2 GU system toxicities, and none experienced grade 3 GU adverse events. The incidence rate of late side effects was similar to that in patients who did not receive SIB treatment for LNs. For example, Zolciak-Siwinska et al [26] reported that $3 \%$ and $6 \%$ of patients experienced grade $3 \mathrm{GU}$ and 3 GI late side effects, respectively. Koh et al [27] reported that $11 \%$ of their patients had grade 3 proctitis, whereas $2 \%$ had grade 3 cystitis; in contrast, Chen et al ${ }^{[28]}$ reported that $4 \%$ of their patients developed grade 3 late GI toxicity, whereas 5.6\% developed grade 3 late GU system toxicity.

In this study, no significant relationship was observed between the volumes of the small bowel that received 62.5 or $60 \mathrm{~Gy}$ in patients with acute GI toxicity. To date, few studies have reported the dose-volume effect relationships for the small bowel; hence, the optimal limited dose for this organ requires further study. Additionally, because the rectal volume receiving 62.5 or 60 Gy is small, no significant relationship was observed between rectum LSEs and the mean rectal volume.

To our knowledge, relatively few studies have investigated the efficacy of SIB-IMRT for positive LNs (which are limited to treatment with CT-guided brachytherapy) or the toxicity to OARs. A limitation of this study, however, is its retrospective nature. Thus, a prospective investigation with a larger sample size is required to definitively confirm the safety and efficacy of this therapeutic approach.

\section{Conclusions}

We demonstrated that a dose of $62.5 \mathrm{~Gy}\left(\mathrm{EQD}_{2}=\right.$ 65 Gy) for positive LNs in patients with locally advanced cervical cancer can achieve excellent clinical outcomes. The toxicity profile when using the IMRT-based SIB technique is acceptable and is comparable with that of previous studies.

\section{Supplementary Material}

Supplementary figures.

http://www.jcancer.org/v10p1103s1.pdf

\section{Acknowledgements}

This study was funded by National Natural Science Foundation of China (grant number 81272346).

\section{Competing Interests}

The authors have declared that no competing interest exists. 


\section{References}

1. Parkin DM, Bray MF, Ferlay MJ, et al. Global cancer statistics, 2002. CA Cancer J Clin. 2005; 55(2): 74-108

2. Goff BA, Muntz HG, Paley PJ, et al. Impact of surgical staging in women with locally advanced cervical cancer. Gynecol Oncol. 1999; 74(3): 436-442.

3. Eifel PJ, Morris M, Wharton JT, et al. The influence of tumor size and morphology on the outcome of patients with FIGO stage IB squamous cell carcinoma of the uterine cervix. Int J Radiat Oncol Biol Phys. 1994; 29(1): 9-16.

4. Tseng JY, Yen MS, Twu NF, et al. Prognostic nomogram for overall survival in stage IIB-IVA cervical cancer patients treated with concurrent chemoradiotherapy. Am J Obstet Gynecol. 2010; 202(2): 174e1-e7.

5. Endo D, Todo Y, Okamoto K, et al. Prognostic factors for patients with cervical cancer treated with concurrent chemoradiotherapy: a retrospective analysis in a Japanese cohort. J Gynecol Oncol. 2015; 26(1): 12-18.

6. Parker K, Gallop-Evans E, Hanna L, et al. Five years' experience treating locally advanced cervical cancer with concurrent chemoradiotherapy and high-dose-rate brachytherapy: results from a single institution. Int J Radiat Oncol Biol Phys. 2009; 74(1): 140-146.

7. Li X, Wei LC, Zhang Y, et al. The prognosis and risk stratification based on pelvic lymph node characteristics in patients with locally advanced cervical squamous cell carcinoma treated with concurrent chemoradiotherapy. Int J Gynecol Cancer. 2016; 26(8): 1472-1479.

8. Berman ML, Keys H, Creasman W, et al. Survival and patterns of recurrence in cervical cancer metastatic to periaortic lymph nodes (a gynecologic oncology group study). Gynecol Oncol. 1984; 19(1): 8-16.

9. Piver MS, Barlow JJ and Krishnamsetty R. Five-year survival (with no evidence of disease) in patients with biopsy-confirmed aortic node metastasis from cervical carcinoma. Am J Obstet Gynecol. 1981; 139(5): 575-578.

10. Gaffney DK, Ericksonwittmann BA, Jhingran A, et al. ACR appropriateness criteria on advanced cervical cancer expert panel on radiation oncology-gynecology. Int J Radiat Oncol Biol Phys. 2011; 81(3): 609-614.

11. Beadle BM, Jhingran A, Yom SS, et al. Patterns of regional recurrence after definitive radiotherapy for cervical cancer. Int J Radiat Oncol Biol Phys. 2010; 76(5): 1396-1403.

12. Liu Y, Yu J, Qian L, et al. Extended field intensity-modulated radiotherapy plus concurrent nedaplatin treatment in cervical cancer. Oncol Lett. 2016; 11(5): 3421-3427.

13. Takuro A, Takafumi T, Goro K, et al. External beam boost irradiation for clinically positive pelvic nodes in patients with uterine cervical cancer. J Radiat Res. 2013; 54(4): 690-696.

14. Mell LK, Sirák I, Wei L, et al. Bone marrow-sparing intensity modulated radiation therapy with concurrent cisplatin for stage IB-IVA cervical cancer: an international multicenter phase II clinical trial (INTERTECC-2).[J]. Int J Radiat Oncol Biol Phys. 2017, 97(3):536-545.

15. Viswanathan AN, Dimopoulos J, Kirisits C, et al. Computed tomography versus magnetic resonance imaging-based contouring in cervical cancer brachytherapy: results of a prospective trial and preliminary guidelines for standardized contours. Int J Radiat Oncol Biol Phys. 2007; 68(2): 491-498.

16. Van LE. Recommendations from gynaecological (GYN) GEC-ESTRO working group (I): concepts and terms in 3D image based 3D treatment planning in cervix cancer brachytherapy with emphasis on MRI assessment of GTV and CTV. Radiother Oncol. 2005; 74(3): 235-245.

17. Hellebust TP, Kirisits C, Berger D, et al. Recommendations from gynaecological (GYN) GEC-ESTRO working group: considerations and pitfalls in commissioning and applicator reconstruction in 3D image-based treatment planning of cervix cancer brachytherapy. Radiother Oncol. 2010; 96(2): 153-160

18. Fowler JF. The linear-quadratic formula and progress in fractionated radiotherapy. Brit J Radiol. 1989; 62(740): 679-694.

19. Yoon MS, Ahn SJ, Nah BS, et al. Metabolic response of lymph nodes immediately after RT is related with survival outcome of patients with pelvic node-positive cervical cancer using consecutive $\left[{ }^{18} \mathrm{~F}\right]$ fluorodeoxyglucose-positron emission tomography/computed tomography. Int J Radiat Oncol Biol Phys. 2012; 84(4): 491-497.

20. Jr WS, Winter K, Levenback C, et al. Extended-field irradiation and intracavitary brachytherapy combined with cisplatin chemotherapy for cervical cancer with positive para-aortic or high common iliac lymph nodes: results of ARM 1 of RTOG 0116. Int J Radiat Oncol Biol Phys. 2007; 68(4): 1081-1087.

21. Rash DL, Lee YC, Kashefi A, et al. Clinical response of pelvic and para-aortic lymphadenopathy to a radiation boost in the definitive management of locally advanced cervical cancer. Int J Radiat Oncol Biol Phys. 2013; 87(2): 317-322.

22. Cihoric N, Tapia $C$, Krüger $K$, et al. IMRT with ${ }^{18}$ FDG-PET $\backslash C T$ based simultaneous integrated boost for treatment of nodal positive cervical cancer. Radiat Oncol. 2014; 9(1): 83-90.

23. Grigsby PW, Singh AK, Siegel BA, et al. Lymph node control in cervical cancer. Int J Radiat Oncol Biol Phys. 2004; 59(3): 706-712.

24. Forrest J, Presutti J, Davidson M, et al. A dosimetric planning study comparing intensity-modulated radiotherapy with four-field conformal pelvic radiotherapy for the definitive treatment of cervical carcinoma. Clin Oncol. 2012; 24(4): 63-70.

25. Portelance L, Chao KS, Grigsby PW, et al. Intensity-modulated radiation therapy (IMRT) reduces small bowel, rectum, and bladder doses in patients with cervical cancer receiving pelvic and para-aortic irradiation. Int J Radiat Oncol Biol Phys. 2001; 51(1): 261-266.

26. Zolciaksiwinska A, Gruszczynska E, Bijok M, et al. Computed Tomography -planned high-dose-rate brachytherapy for treating uterine cervical cancer. Int J Radiat Oncol Biol Phys. 2016; 96(1): 87-92.

27. Koh V, Choo BA, Lee KM, et al. Feasibility study of toxicity outcomes using GEC-ESTRO contouring guidelines on CT based instead of MRI-based planning in locally advanced cervical cancer patients. Brachytherapy. 2017; 16(1): $126-132$.

28. Chen CC, Wang L, Lin JC, et al. The prognostic factors for locally advanced cervical cancer patients treated by intensity-modulated radiation therapy with concurrent chemotherapy. J Formos Med Assoc. 2015; 114(3): 231-237. 